\title{
Single Stage Surgery for Open Lisfranc Injury: A Case Report
}

\author{
Rajesh B Lakhey, Prawesh S Bhandari, Govinda KC
}

\author{
Author(s) affiliation \\ 'Department of Orthopedics and \\ Trauma Surgery, Maharajgunj \\ Medical Campus, Tribhuvan \\ University Teaching Hospital, \\ Institute of Medicine, Maharajgunj, \\ Kathmandu, Nepal

\section{Corresponding author} \\ Rajesh B Lakhey, MBBS, FCPS \\ rajeshlakhey@gmail.com
}

\begin{abstract}
Management of open Lisfranc injuries is challenging. The improper management of the injuries might lead to bad results like skin necrosis and arthritis of Lisfranc's joints. A case of Myerson type A Gustilo Grade IIIB open Lisfranc injury was managed with single stage debridement and internal fixation. At three years follow-up, the result was excellent with American Orthopedic Foot and Ankle Society (AOFAS) midfoot score of 100 and the patient had rejoined his previous occupation requiring prolonged walking.
\end{abstract}

\section{Keywords}

Open Lisfranc injury, single stage internal fixation

\section{Submitted}

Jan 10, 2021

\section{Accepted}

Mar 16, 2021

\section{INTRODUCTION}

isfranc injuries account for $0.2 \%$ of all fractures. ${ }^{1}$ The reported incidence of this uncommon injury is approximately 1 per 55,000 persons per year. ${ }^{2}$ High energy open Lisfranc injuries mainly result from being crushed under a heavy object, traffic accidents, or falling from a height and are represented by severe Lisfranc joint fracturedislocation with serious soft tissue injuries. ${ }^{3}$ A case which presented with Gustilo grade IIIB open and Myerson type A Lisfranc injury was managed with single stage debridement and internal fixation.

\section{CASE PRESENTATION}

A 24 years old gentleman presented with Myerson type A Gustilo grade IIIB open Lisfranc injury of right foot to Tribhuvn University Teaching Hospital in a road traffic accident, being involved in high speed motorbike accident. (Figure 1) One stage debridement of the injury with anatomical reduction through the wound on the medial aspect of the foot; an incision on the lateral aspect of the dorsum of the foot, and fixation of the Lisfranc injury was done. A partially threaded screw was passed from medial cuneiform to the base of second metatarsal, two K-wires were passed from the base second metatarsal to the middle cuneiform, another K-wire from the third metatarsal to the lateral cuneiform, and one K-wire was passed from base of fourth and fifth 


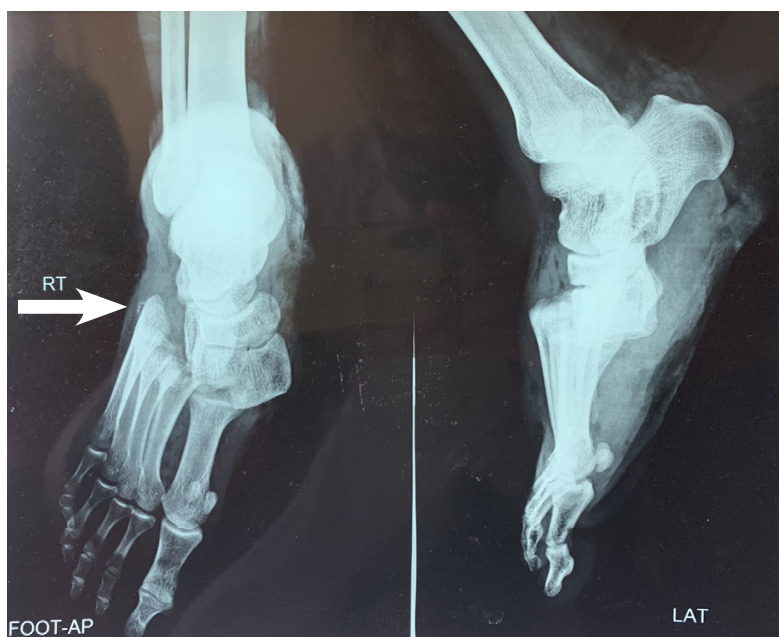

Fig 1. X-ray at injury showing Myerson Type A Lisfranc fracture dislocation

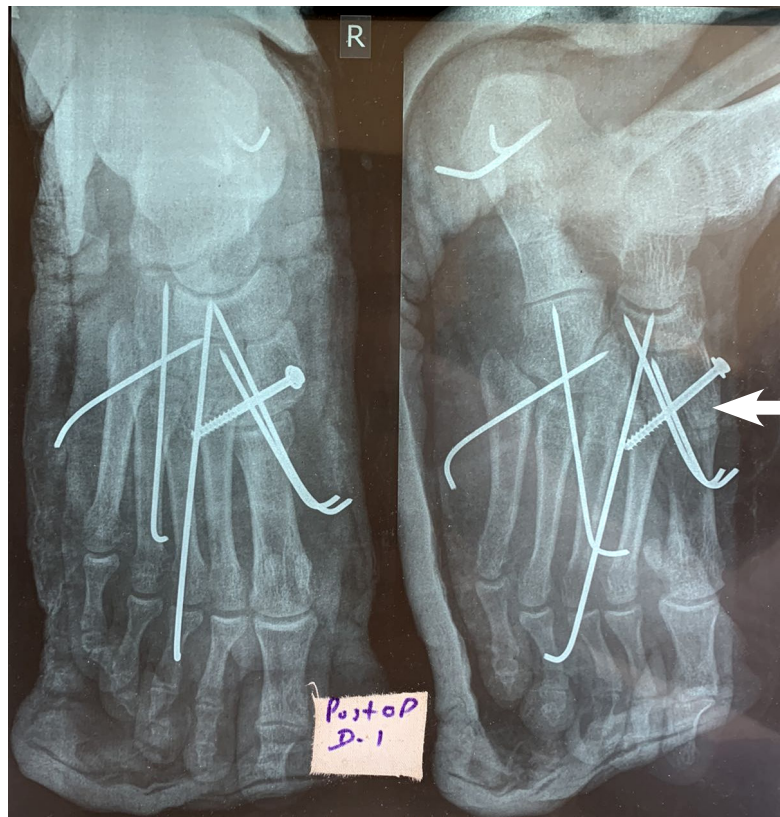

Fig 2. Post-operative X-ray showing well-reduced Lisfranc joint

metatarsals to the cuboid each. The heel flap was fixed to the calcaneum with two K-wires.(Figure 2) The open wound on the medial side of the foot was loosely closed. There was no infection in the wound. After development of the granulation tissue, skin graft was done. Post-operative slab support was applied. K-wires were removed after 3 months and weight bearing was started. The screw was removed 4 months post-operatively.

At 3 years follow-up the American Orthopedic Foot and Ankle Society (AOFAS) midfoot score was 100 , with only occasional morning stiffness in foot lasting for a few minutes (Figure 3,4). The patient has rejoined his previous profession of policeman requiring prolonged walking and walking on uneven surfaces.

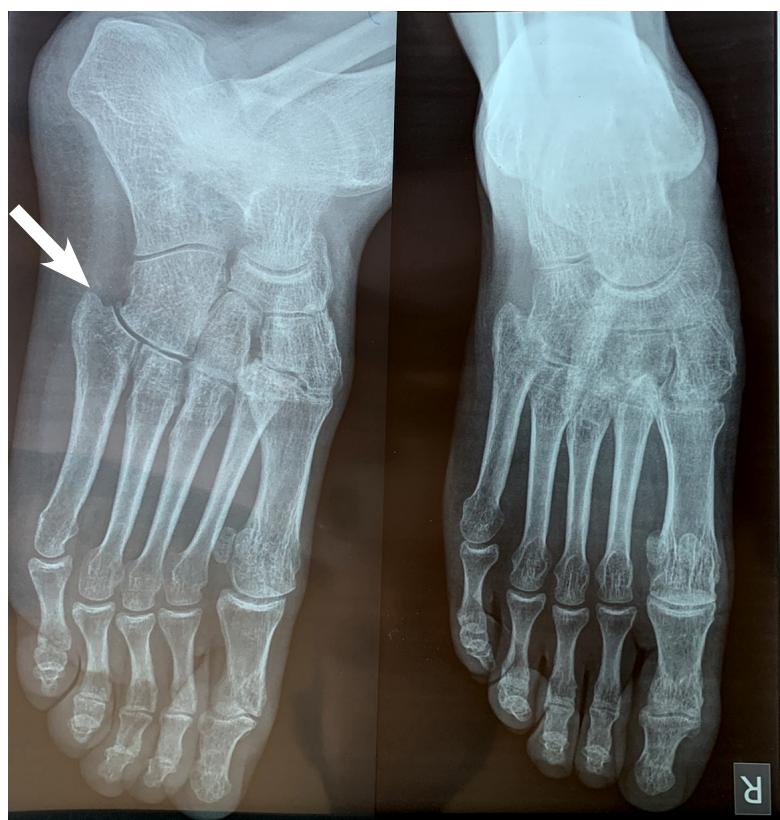

Fig 3. X-ray at 3 years followup showing well-reduced Lisfranc Joint with no features of arthritis
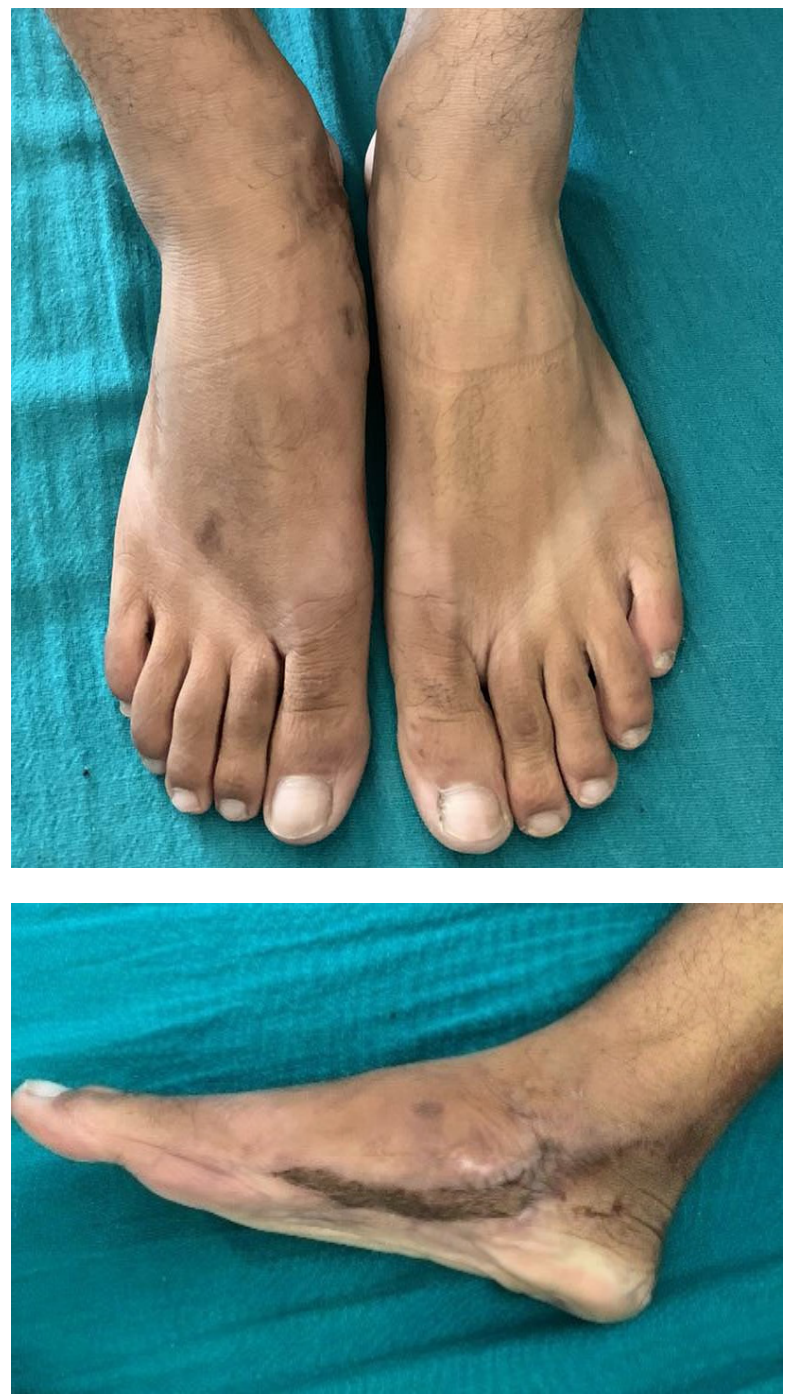

Fig 4. Clinical photograph at 3 years follow-up showing normal looking plantigrade foot 


\section{DISCUSSION}

The difficulty in the clinical treatment of open Lisfranc fracture and dislocation results from the severe destruction of the bony structure, with poor condition of local soft tissue. Thus, staged treatment has been frequently performed ${ }^{4,5}$, with initial external fixation followed by definitive fixation. However it is practical to attempt a safe and effective one-stage definitive internal fixation owing to the fact that the two stage procedure would require long incisions for reductions due to scarring and may even require osteotomies and fusions. ${ }^{6}$ In our case, we did single stage reduction and fixation with excellent result.

Proper debridement and wound care resulted in good soft tissue healing in our case. The results were as good as that on using vaccum suction drainage system, which is common in developed countries $^{7}$ but unavailable in our part of the world due to financial issues.

The excellent result of the surgery may be due to the anatomical reduction of the joints, which was carefully done from the medial open wound and by a longitudinal incision on the lateral aspect of the dorsum of the foot. Previous studies demonstrated that malreduction during surgery led to poor function and imaging scores in upto $49.6 \%$ of the cases. Nithyananth ${ }^{3}, \mathrm{Kuo}^{6}$ and Kadow ${ }^{7}$ demonstrated the standard to ensure anatomical reduction during the surgery and we followed the guidelines.

Lisfranc injuries have been treated with one stage joint fusions too $^{8}$, but some researchers demonstrated no statistically significant difference between cases treated with one-stage joint fusion and those treated with one-stage internal fixation, after a long-term follow-up. ${ }^{9}$ Joint fusions could be done later on for symptomatic Lisfranc joint instability. ${ }^{6}$ Our case had excellent result with single stage internal fixation and does not have symptomatic Lisfranc joint instability in the followups.

\section{CONCLUSION}

When managing Lisfranc fracture dislocation, the goal should be to achieve a painless, functional normal-looking plantigrade foot. The Myerson type A and Grade IIIB open Lisfranc injury was managed by single stage debridement and internal fixation with excellent results.

\section{CONSENT}

Informed consent was was obtained from the patient for publication of this case report.

\section{CONFLICT OF INTEREST}

None declared.

\section{REFERENCES}

1. Hardcastle PH, Reschauer R, Kutscha-Lissberg E et al. Injuries to the tarsometatarsal joint: Incidence, classification and treatment. J Bone Joint Surg Br 1982;64(3):349-356.

2. Mantas JP, Burks RT. Lisfranc injuries in the athlete. Clin Sports Med 1994;13(4):719-730.

3. Nithyananth M, Boopalan PR, Titus VT et al. Long-term outcome of high-energy open Lisfranc injuries: a retrospective study. J Trauma. 2011;70:710-6.

4. Coetzee JC, Ly TV. Treatment of primarily ligamentous Lisfranc joint injuries: primary arthrodesis compared with open reduction and internal fixation. Surgical technique. J Bone Joint Surg Am. 2007;89:5122-7.

5. Qu W, Ni S, Wang Z et al. Severe open Lisfranc injuries: onestage operation through internal fixation associated with vacuum sealing drainage. J Orthop Surg Res 2016; 11:134.

6. Kuo RS, Tejwani NC, Digiovanni CW, et al. Outcome after open reduction and internal fixation of Lisfranc joint injuries. J Bone Joint Surg Am. 2000;82-A:1609-18.

7. Kadow TR, Siska PA, Evans AR et al. Staged treatment of high energy midfoot fracture dislocations. Foot Ankle Int. 2014;35:1287-91.

8. Henning JA, Jones $C B$, Sietsema $D L$ et al. Open reduction internal fixation versus primary arthrodesis for Lisfranc injuries: a prospective randomized study. Foot Ankle Int. 2009;30:913-22.

9. Seybold JD, Coetzee JC. Lisfranc injuries: when to observe, fix, or fuse. Clin Sports Med. 2015;34:705-23. 